\title{
The Role of Water in the Photocatalytic Degradation of Acetonitrile and Toluene in Gas-Solid and Liquid-Solid Regimes
}

\author{
Maurizio Addamo, ${ }^{1}$ Vincenzo Augugliaro, ${ }^{1}$ Salvatore Coluccia, ${ }^{2}$ Agatino Di Paola, ${ }^{1}$ Elisa García-López, ${ }^{1}$ \\ Vittorio Loddo, ${ }^{1}$ Giuseppe Marci, ${ }^{1}$ Gianmario Martra, ${ }^{2}$ and Leonardo Palmisano ${ }^{1}$ \\ ${ }^{1}$ Dipartimento di Ingegneria Chimica dei Processi e dei Materiali, Università degli Studi di Palermo, Viale delle Scienze, \\ 90128 Palermo, Italy \\ ${ }^{2}$ Dipartimento di Chimica IFM and NIS Centre of Excellence, Università degli Studi di Torino, Via Pietro Giuria 7, \\ 10125 Torino, Italy
}

Received 25 June 2005; Revised 17 August 2005; Accepted 20 September 2005

\begin{abstract}
Photocatalytic degradation of acetonitrile and toluene was carried out both in gas-solid and in liquid-solid regimes by using commercial $\mathrm{TiO}_{2}$ samples (Merck and Degussa P25). The investigation was mainly aimed to study the influence of water present in the reaction environment on the mechanism and degradation rate of two probe molecules. In gas-solid regime, the reacting mixture consisted of toluene or acetonitrile, oxygen, nitrogen, and water vapour. The main degradation product of toluene was $\mathrm{CO}_{2}$ with small amounts of benzaldehyde. In the presence of water vapour, the activity of $\mathrm{TiO}_{2}$ Merck remained stable but greatly decreased if water was absent. $\mathrm{TiO}_{2}$ Degussa P25 continuously deactivated, even in the presence of water vapour. With both catalysts, the photodegradation products of acetonitrile were $\mathrm{CO}_{2}$ and $\mathrm{HCN}$; the activity was stable and was independent of the presence of water vapour in the reacting mixture. The production of HCN represents a drawback of acetonitrile photocatalytic degradation but the elimination of $\mathrm{HCN}$ is not actually a problem. In liquid-solid regime, the main intermediates of toluene photodegradation were $p$-cresol and benzaldehyde; traces of pyrogallol and benzyl alcohol were also found. Benzoic acid, hydroquinone, and trans, trans muconic acid were detected only when $\mathrm{TiO}_{2}$ Merck was used. The photodegradation products of acetonitrile were cyanide, cyanate, formate, nitrate, and carbonate ions.
\end{abstract}

Copyright (c) 2006 Maurizio Addamo et al. This is an open access article distributed under the Creative Commons Attribution License, which permits unrestricted use, distribution, and reproduction in any medium, provided the original work is properly cited.

\section{INTRODUCTION}

Advanced oxidation processes (AOPs) of organic pollutants have been extensively studied especially with the aim of environment remediation. Among them, heterogeneous photocatalysis in the presence of irradiated polycrystalline semiconductors has shown to be a viable alternative for treating contaminated gaseous and aqueous streams [1-5]. In particular, photocatalytic oxidation offers various advantages compared with traditional treatment methods because the photoreactions occur at room temperature and atmospheric pressure, under radiation of the near-UV region and at measurable rates also for very low concentrations of reactants [6]. Toluene is a widespread chemical used for the preparation of many compounds of industrial interest such as benzaldehyde, benzyl alcohol, benzoic acid, chloro derivatives, and so forth, so that it can be found in many industrial waste effluents. It is a very noxious compound and many strategies have been identified to reduce its presence in the environment. Acetonitrile is an extremely stable and toxic molecule present in civil and industrial wastewaters. It is widely used as eluent for HPLC analyses, and air in contact with liquid acetonitrile can be easily contaminated owing to the high volatility of the last. Toluene and acetonitrile are very interesting molecules for photooxidation studies; toluene is a molecule representative of aromatic compounds whilst acetonitrile is a Lewis base with an alkyl and a cyanide group that may undergo different oxidation routes.

Heterogeneous photocatalysis has been already used for toluene abatement and previous papers report the partial or complete oxidation of toluene both in gas-solid [7-12] and in liquid-solid systems [13-17]. Ibusuki and Takeuchi [7] found that the presence of water vapour was beneficial in order to achieve the almost complete mineralization of toluene by carrying out the photooxidation of toluene vapour on $\mathrm{TiO}_{2}$ at room temperature. Obee and Brown [8] studied the 


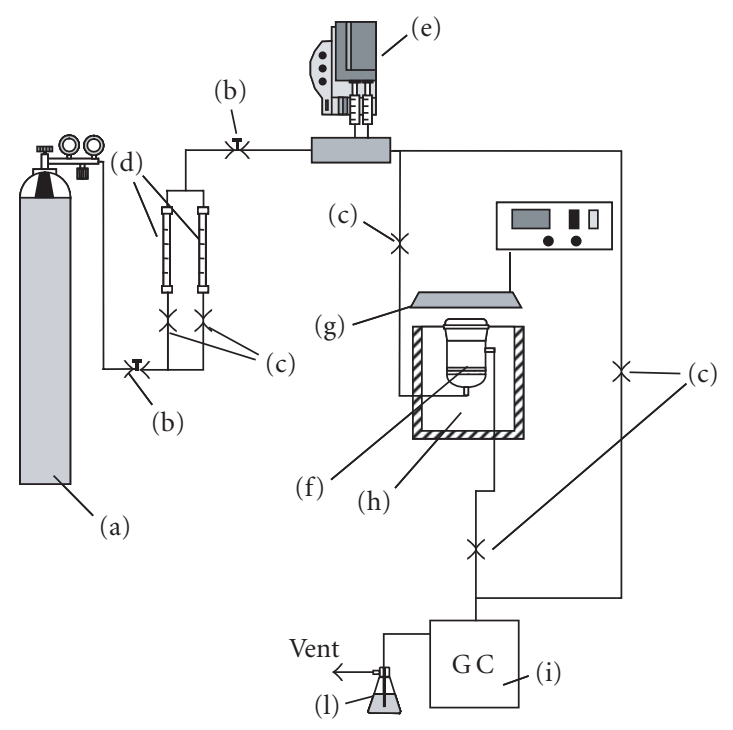

FIgURE 1: Experimental setup used for toluene photocatalytic degradation in gas-solid regime: (a) air cylinder; (b) control valves; (c) switch valves; (d) rotameters; (e) infusion pump; (f) photoreactor; (g) irradiation system; (h) oven; (i) gas chromatograph; (j) $\mathrm{CO}_{2}$ trap.

influence of the competitive adsorption of water and toluene vapours on the photooxidation rate. Luo and Ollis [9] and Einaga et al. [10] reported that the influence of water is complex and an optimum water vapour concentration was found at which a maximum of reactivity was observed. Fujihira et al. $[13,14]$ studied the photocatalytic oxidation of toluene in aqueous aerated suspensions; they reported the formation of cresols, benzaldehyde, and benzyl alcohol, depending on the $\mathrm{pH}$ of the solution and on the used photocatalyst. Navio et al. [15] confirmed the formation of benzaldehyde. Recently the toluene photooxidation has been carried out in gas-solid $[11,12]$ or liquid-solid $[16,17]$ regimes. The influence of water on the product distribution was investigated and the performances of photooxidation process in both regimes were compared.

Lichtin et al. [18] firstly reported the feasibility of the photocatalytic oxidation of acetonitrile both in liquid and in vapour phases. Acetonitrile showed to be much more reactive in gas phase than in liquid phase. In gas-solid regime they found cyanogen, indicating the formation of cyanide radicals and their subsequent dimerization. Zhuang et al. [19] reported that the final oxidation products were $\mathrm{CO}_{2}, \mathrm{H}_{2} \mathrm{O}$, and $\mathrm{CO}_{3}{ }^{2-}$. Isocyanate was also found adsorbed onto the catalyst surface; its presence is considered a clue of the formation of free cyanide. The photocatalytic oxidation of acetonitrile was also carried out in aqueous suspensions irradiated by sunlight [20]. Acetonitrile was oxidised to carbonate and nitrate and the main inorganic intermediate detected was cyanate, while only traces of cyanide and nitrite were found. Recently the kinetics and mechanistic aspects of acetonitrile photocatalytic degradation in gas-solid regime have been investigated by using $\mathrm{TiO}_{2}$ Degussa P25 and $\mathrm{TiO}_{2}$ Merck [21]. With both

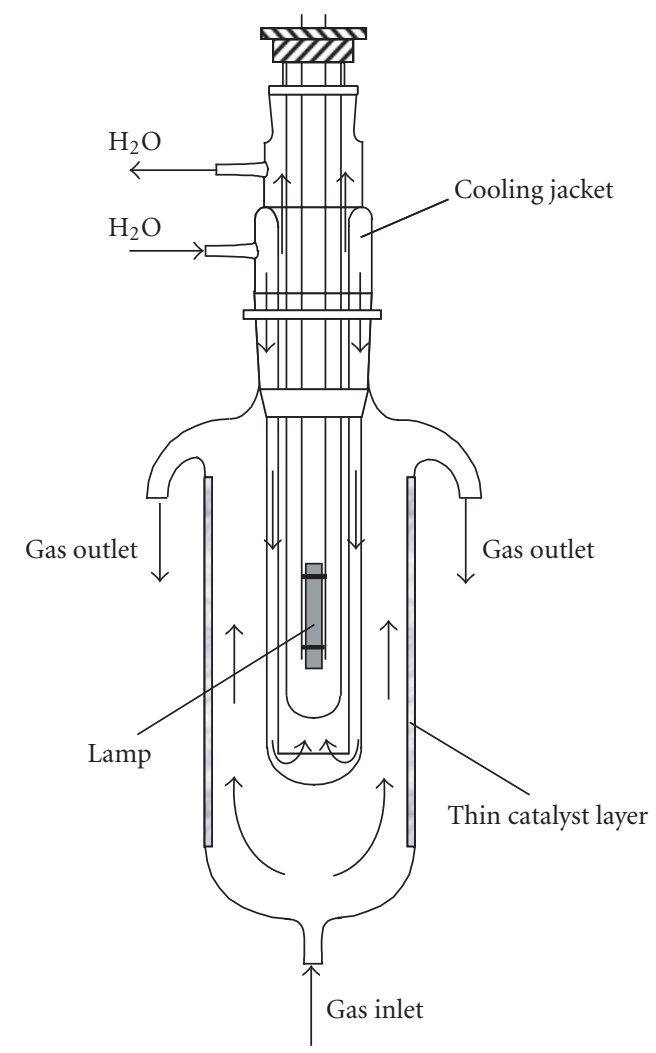

FIGURE 2: Continuous annular photoreactor for acetonitrile photocatalytic degradation in gas-solid regime.

catalysts, the photodegradation products of acetonitrile were $\mathrm{CO}_{2}$ and $\mathrm{HCN}$; the presence of $\mathrm{HCN}$ is a drawback for the application of photocatalytic method to acetonitrile degradation since a further step devoted to the elimination of $\mathrm{HCN}$ must be added to the photoprocess.

This work has been focused on elucidating the role of water present in the reaction environment for the photocatalytic degradation of toluene or acetonitrile. To this aim, the degradation runs have been carried out both in gas-solid and in liquid-solid regime. The information obtained from the photoreactivity experiments, together with that obtained by previous FT-IR spectroscopy investigations [21], has been used for clarifying (at least from a macroscopic point of view) the influence of water phase, liquid or gaseous, on the photoreaction performance and to check whether its influence depends, or not, on the chemical features of the molecules undergoing the photoreaction process.

\section{EXPERIMENTAL}

All the photoreactivity runs were carried out by using two commercial $\mathrm{TiO}_{2}$ samples: Merck (100\% anatase, BET specific surface area: ca. $10 \mathrm{~m}^{2} \cdot \mathrm{g}^{-1}$ ) and Degussa P25 (ca. $80 \%$ anatase and $20 \%$ rutile, BET specific surface area: ca. $\left.50 \mathrm{~m}^{2} \cdot \mathrm{g}^{-1}\right)$. The powders used in the photoreactivity runs did not undergo any preliminary treatment. 


\subsection{Gas-solid regime}

Figure 1 shows the setup used for carrying out the reactivity runs. For toluene degradation, a continuous Pyrex photoreactor with cylindrical shape (internal diameter: $5 \mathrm{~cm}$; height: $10 \mathrm{~cm}$ ) was used. A porous glass septum on the bottom of the cylinder allowed to sustain the fixed bed of photocatalyst and to distribute the inlet gaseous mixture. The photoreactor was vertically positioned inside a thermostatted chamber and it was irradiated from the top by a $400 \mathrm{~W}$ medium pressure $\mathrm{Hg}$ lamp (Polymer GN ZS, Helios Italquartz) placed at a distance of $12 \mathrm{~cm}$ from the top of the catalyst bed. The irradiated surface of the bed was $19.6 \mathrm{~cm}^{2}$. The radiant energy impinging on the reactor, measured at $\lambda=360 \mathrm{~nm}$ by using a radiometer UVX Digital, was $4.5 \mathrm{~mW} \cdot \mathrm{cm}^{-2}$. The temperature inside the photoreactor was maintained at ca. $403 \pm 2 \mathrm{~K}$. The gas flow rates ranged from 0.05 to $0.25 \mathrm{~cm}^{3} \cdot \mathrm{s}^{-1}$. Toluene and water concentrations in the feed were 0.03 and $0.75 \mathrm{mM}$, respectively. The reactivity runs were carried out at equal total surface area of photocatalyst $\left(100 \mathrm{~m}^{2}\right)$ by using $10 \mathrm{~g}$ of $\mathrm{TiO}_{2}$ Merck or $2 \mathrm{~g}$ of $\mathrm{TiO}_{2}$ Degussa P25. The corresponding fixed bed heights were ca. 1 and $2 \mathrm{~cm}$, respectively.

For acetonitrile degradation, a continuous Pyrex annular photoreactor (see Figure 2) was used. The photoreactor was provided with ports for the inlet and outlet of the reacting mixture. A $500 \mathrm{~W}$ medium pressure $\mathrm{Hg}$ lamp (Helios Italquartz, Italy) was located in the internal tube of annulus. A cooling jacket with circulating water surrounded the lamp. The photon flux emitted by the lamp was measured using a radiometer (UVX Digital) leaned against the external wall of the photoreactor. The mean value was $19.0 \mathrm{~mW} \cdot \mathrm{cm}^{-2}$. A thin layer of catalyst ( $1 \mathrm{~g}$ for both photocatalysts) covered the internal wall of the outer tube of annulus. In these conditions, the light transmitted by the $\mathrm{TiO}_{2}$ layer was negligible, as checked with the radiometer. The reacting mixture consisted of acetonitrile, oxygen, nitrogen, and water; the concentration ranges of acetonitrile, oxygen, and water vapour were $0.1-0.8,5-40$, and $0-0.3 \mathrm{mM}$, respectively. For the majority of runs, the gas flow rate was $1.87 \mathrm{~cm}^{3} \cdot \mathrm{s}^{-1}$. The temperature of the photoreactor was $303 \pm 2 \mathrm{~K}$ at steady-state conditions.

The procedure of the photoreactivity runs was always the same. The gas fed to the photoreactor was obtained by adding liquid toluene or acetonitrile and water by means of an infusion pump (Orion sage pump $\mathrm{M} 361$ ) to $\mathrm{N}_{2}-\mathrm{O}_{2}$ mixtures. The irradiation started only when steady-state conditions were achieved. The runs lasted $25 \mathrm{~h}$ for toluene degradation and $4 \mathrm{~h}$ for acetonitrile. The gas at the exit of the photoreactor was periodically analysed by an online gas chromatograph (Hewlett-Packard 6890) equipped with an FID detector and an Alltech Carbograph column (2 $\mathrm{m}$ long $\times$ $2 \mathrm{~mm}$ i.d.) for toluene analysis or a methyl siloxane column $\left(30 \mathrm{~m} \times 320^{-} \mathrm{m} \times 0.25^{-} \mathrm{m}\right.$ film thickness, Hewlett-Packard HP-1) for acetonitrile analysis. For toluene runs, the gas at the outlet of the photoreactor was continuously bubbled in a trap containing pure water while for acetonitrile runs the trap contained an aqueous solution of sodium hydroxide $(0.1 \mathrm{M})$. The trap solution was analysed at the end of the run by HPLC and/or ionic chromatography. The HPLC was a Varian 9010 Solvent Delivery System Pump coupled with a Varian 9050 variable wavelength UV-Vis detector; an Alltech Alltima column $\mathrm{C}_{18} 5^{-} \mathrm{m}$ ( $250 \mathrm{~mm}$ long $\times 4.6 \mathrm{~mm}$ i.d. $)$ was used. The eluent was a mixture methanol $/ \mathrm{H}_{3} \mathrm{PO}_{4}(0.05 \%$ in water), 45/55 v/v. The quantitative determination of anionic species contained in the trap was carried out by using an ionic chromatograph (Dionex DX 120) equipped with an Ion Pac AS14 $4 \mathrm{~mm}$ column (250 mm long, Dionex). The eluent was an aqueous solution of $\mathrm{NaHCO}_{3}(8 \mathrm{mM})$ and $\mathrm{Na}_{2} \mathrm{CO}_{3}$ $(1 \mathrm{mM})$ at a flow rate of $1.67 \cdot 10^{-2} \mathrm{~cm}^{3} \cdot \mathrm{s}^{-1}$. The quantitative determination of cyanide ion was performed by an ion sensitive electrode (Orion model 94-06) in an expandable ion analyser (Orion EA 920). In order to perform a mass balance on carbon for toluene and acetonitrile degradation, once the photoreactivity reached steady-state conditions, the gaseous mixture leaving the photoreactor was bubbled for a fixed interval of time in a saturated aqueous solution of barium hydroxide. The total amount of produced $\mathrm{CO}_{2}$ was determined as $\mathrm{BaCO}_{3}$. At the end of each degradation run, the catalyst was scraped from the photoreactor wall and put in water in the case of acetonitrile, while it was washed with acetonitrile in the case of toluene. After separation of the solid by filtration through $0.45^{-} \mathrm{m}$ cellulose acetate filter (HA, Millipore), the resulting solution was analysed by HPLC and the ionic chromatograph previously described. All the reagents were analytical grade (Aldrich).

\subsection{Liquid-solid regime}

A Pyrex batch photoreactor of cylindrical shape containing 1.5 litres of aqueous suspension was used for performing all the photoreactivity experiments. The photoreactor was provided with ports in its upper section for the inlet and outlet of gases, for sampling and for $\mathrm{pH}$ and temperature measurements. A $500 \mathrm{~W}$ medium pressure Hg lamp (Helios Italquartz) was immersed within the photoreactor; the lamp was cooled by water circulating through a Pyrex jacket and the suspension had a temperature of about $300 \mathrm{~K}$. The radiation energy impinging on the suspension had an average value of $19.0 \mathrm{~mW} \cdot \mathrm{cm}^{-2}$ for both toluene and acetonitrile photocatalytic experiments. For all the runs, the catalyst amount was $0.4 \mathrm{~g} \cdot \mathrm{dm}^{-3}$. A magnetic stirrer guaranteed a satisfactory suspension of the photocatalyst and the uniformity of the reacting mixture. Pure oxygen at atmospheric pressure was continuously bubbled in the suspension during the course of the photocatalytic runs; the bubbling started $0.5 \mathrm{~h}$ before switching on the lamp. Samples for analyses were withdrawn at fixed intervals of time and the catalyst was immediately separated by filtration. The runs lasted from 1.5 to $10 \mathrm{~h}$. The initial $\mathrm{pH}$ of the suspension was 6.0 for toluene and 11.0 for acetonitrile degradation. Initial toluene concentrations were in the $0.1-1.4 \mathrm{mM}$ range, being the highest concentration below the solubility limit of toluene in water at room temperature $(5.4 \mathrm{mM})$. The initial acetonitrile concentration was varied in the $0.24-24.00 \mathrm{mM}$ range.

Toluene and its intermediate oxidation products were analysed by HPLC using two different Alltech columns. Toluene analysis was carried out with an Econosphere C18 
$3^{-} \mathrm{m}$ column, $(150 \mathrm{~mm}$ long $\times 4.6 \mathrm{~mm}$ i.d. $)$ with an acetonitrile/water $(35 / 65 \mathrm{v} / \mathrm{v})$ eluent while the oxidation products of toluene were analysed with an Econosil C18 $10^{-} \mathrm{m}$ column $(250 \mathrm{~mm}$ long $\times 4.6 \mathrm{~mm}$ i.d. $)$ using an acetonitrile/water $(65 / 35 \mathrm{v} / \mathrm{v})$ eluent.

The quantitative determination of acetonitrile was routinely performed with the same gas chromatograph, column, and conditions used for the gas-solid experiments. $1 \mathrm{~mL}$ sample was placed in a vial and the compounds present in the vapour phase were extracted (extraction time, 5 minutes) by using a $75^{-} \mathrm{m}$ Carboxen-PDMS SPME (solid phase microextraction) fibre assembly (Supelco) with a fibre holder for manual sampling. The holder was then placed in the split/splitless injector of the gas chromatograph maintained at $523 \mathrm{~K}$. The temperature of the oven was $310 \mathrm{~K}$ for the first 3 minutes and then it was increased up to $523 \mathrm{~K}$ at a rate of $60 \mathrm{~K} \cdot \mathrm{min}^{-1}$. The quantitative determination of intermediate ionic species was carried out by using a cyanide selective electrode and ionic chromatography in the same way described above for the gas-solid experiments. Total organic carbon (TOC) analyses were carried out for all the runs by using a 5000A Shimadzu total organic carbon analyser in order to check the complete mineralization of toluene and acetonitrile.

\section{RESULTS AND DISCUSSION}

For both model molecules, blank reactivity tests were performed under the same experimental conditions used for the photodegradation experiments but in the absence of catalyst, oxygen, or light. In liquid-solid regime, no reactivity was observed indicating that the contemporary presence of $\mathrm{O}_{2}$, catalyst, and irradiation is needed for the occurrence of toluene and acetonitrile degradation processes; in gas-solid regime the same behaviour was observed both in the presence or absence of water vapour in the mixture fed to the photoreactor.

\subsection{Toluene degradation}

\subsubsection{Gas-solid regime}

Preliminary tests were performed to determine suitable flow rate conditions to avoid mass transport limitations on the photoreaction. To this aim, reactivity experiments were carried out by increasing the flow rate at constant concentration values of toluene and water $(0.03$ and $0.75 \mathrm{mM}$, resp.).

The toluene disappearance rate per unit of catalyst surface area, $\left(-r_{S}\right)$, was determined by applying the following mass balance on the whole photoreactor:

$$
\left(-r_{S}\right)=\frac{W}{S}\left(C_{\mathrm{I}}-C_{\mathrm{O}}\right)
$$

where $W$ is the volumetric gas flow rate, $S$ the total surface area of catalyst, and $C_{\mathrm{I}}$ and $C_{O}$ the inlet and outlet toluene molar concentrations, respectively.

The reaction rate increased for flow rates up to ca. $0.04 \mathrm{~cm}^{3} \cdot \mathrm{s}^{-1}$ whilst, for higher values, it did not change significantly. This finding indicates that the mass transfer resistance limits the reaction rate when the flow rate values

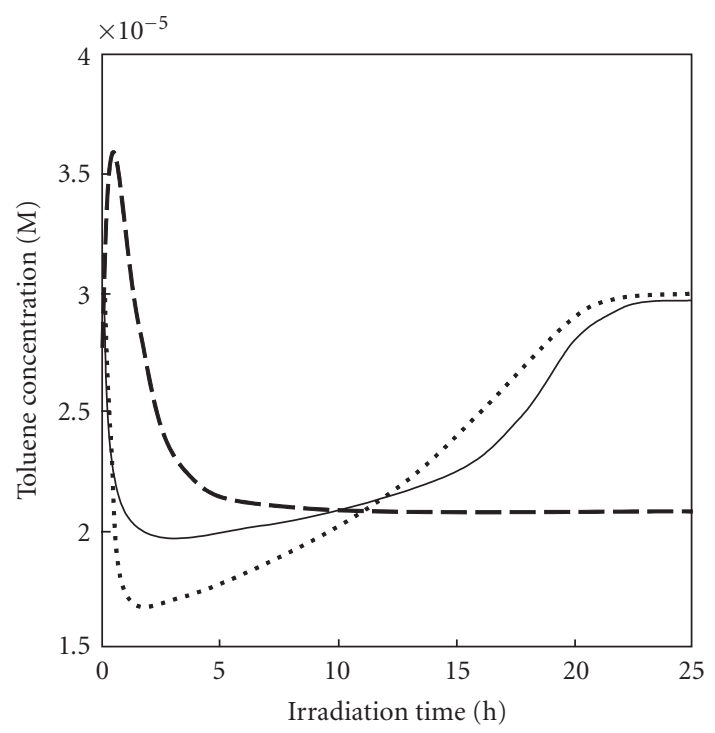

Figure 3: Toluene photocatalytic degradation in gas-solid regime. Experimental results of outlet toluene concentration versus irradiation time by using $\mathrm{TiO}_{2}$ Degussa $\mathrm{P} 25$ in the presence of water $[\cdots]$ and $\mathrm{TiO}_{2}$ Merck in the presence (- - -) or in the absence (-) of water vapour. Flow rate: $0.04 \mathrm{~cm}^{3} \cdot \mathrm{s}^{-1}$; inlet toluene concentration: $0.03 \mathrm{mM}$, water vapour concentration: $0.75 \mathrm{mM}$.

are less than $0.04 \mathrm{~cm}^{3} \cdot \mathrm{s}^{-1}$. Consequently, all the photocatalytic experiments were carried out in the $0.05-0.25 \mathrm{~cm}^{3} \cdot \mathrm{s}^{-1}$ range. In order to check that all the fixed bed was irradiated, that is, the penetration depth of radiation was higher than the height of the fixed bed, a few runs were carried out employing larger amounts of $\mathrm{TiO}_{2}$ Merck catalyst. It was found that the activity increased proportionally to the catalyst amount so that it was concluded that at the usual bed height $(1 \mathrm{~cm})$ all the catalyst particles were irradiated.

Figure 3 reports the values of toluene concentration in the photoreactor outlet versus the irradiation time for three representative runs; for the sake of clarity, in this figure the experimental points have been substituted by interpolating lines. These runs were carried out with the same inlet toluene concentration in the presence of water vapour $\left(\mathrm{TiO}_{2}\right.$ Merck and $\mathrm{TiO}_{2}$ Degussa P25) and in the absence of water vapour $\left(\mathrm{TiO}_{2}\right.$ Merck). The oxidation products detected both in the gas phase and on the photocatalyst surface are reported in Table 1.

For $\mathrm{TiO}_{2}$ Merck, in the presence of water vapour, a rapid increase of outlet toluene concentration after switching on the lamp was observed, followed by a slow decrease down to a steady value. A maximum conversion of ca. $24 \%$, corresponding to an oxidation rate of $2.7 \times 10^{-12} \mathrm{~mol} \cdot \mathrm{s}^{-1} \cdot \mathrm{m}^{-2}$, was achieved at steady state conditions. The main photooxidation products detected in the gas phase were carbon dioxide and benzaldehyde. Benzaldehyde concentration values (not shown in Figure 3) reached a maximum after 3 hours of irradiation and therefore slightly decreased reaching a steady value of $2.5 \cdot 10^{-8} \mathrm{~mol} \cdot 1^{-1}$. For $\mathrm{TiO}_{2}$ Degussa P25, the outlet toluene concentration showed a sharp decrease followed 
TABLe 1: Photocatalytic degradation of toluene. Intermediates detected in liquid-solid regime and in gas-solid regime.

\begin{tabular}{|c|c|c|c|c|}
\hline \multirow[b]{2}{*}{ Intermediates } & \multicolumn{2}{|c|}{$\mathrm{TiO}_{2}$ Merck } & \multicolumn{2}{|c|}{$\mathrm{TiO}_{2}$ Degussa P25 } \\
\hline & Liquid-solid & Gas-solid & Liquid-solid & Gas-solid \\
\hline paracresol (4-hydroxytoluene) & Yes & No & Yes & No \\
\hline Benzaldehyde & Yes & Yes & Yes & Yes \\
\hline Benzyl Alcohol & Yes & Yes $^{*}$ & Yes & Yes $^{*}$ \\
\hline Benzoic Acid & Yes & Yes $^{*}$ & No & Yes $^{*}$ \\
\hline $\begin{array}{c}\text { Pyrogallol } \\
(1,2,3 \text { trihydroxybenzene })\end{array}$ & Yes & No & Yes & No \\
\hline $\begin{array}{c}\text { Hydroquinone } \\
\text { (1,2 dihydroxybenzene) }\end{array}$ & Yes & No & No & No \\
\hline $\begin{array}{c}\text { Trans, trans muconic acid } \\
\text { (trans, trans }-2,4 \text { esadiendioic acid) }\end{array}$ & Yes & No & No & No \\
\hline
\end{tabular}

by a slow increase up to the inlet value thus indicating the occurrence of deactivation. The main oxidation product was carbon dioxide; benzaldehyde was detected in very small quantities while large amounts of benzoic acid were found adsorbed onto the catalyst surface.

In the absence of water vapour, the run carried out with $\mathrm{TiO}_{2}$ Merck showed that water greatly affects the performance of the photocatalyst, which continuously deactivates. Indeed, a maximum conversion of $33.5 \%$ (corresponding to an oxidation rate of $\left.4.2 \cdot 10^{-12} \mathrm{~mol} \cdot \mathrm{s}^{-1} \cdot \mathrm{m}^{-2}\right)$ was reached after ca. 2 hours and after that, the photoreactivity decreased. The initial decrease of outlet toluene concentration, however, was more significant than that observed in the presence of water vapour. This finding can be explained by considering that the absence of water vapour in the reacting mixture favours the adsorption of toluene on the catalyst surface but it hinders the benzaldehyde desorption. In this case, benzaldehyde adsorbed on the catalyst surface can be more easily attacked by oxidant radical species and transformed in stable species that remain adsorbed on the surface. Therefore, the catalyst deactivation in the absence of water may be due to irreversible surface dehydroxylation occurring in the course of toluene oxidation and/or to the formation of products strongly adsorbed, occupying the active surface sites, and hindering the toluene adsorption. A clear clue of the formation of these stable products (probably aromatic oligomers) is that at the end of the run the catalyst exhibited an ochre colour that did not completely disappear even after washing with acetonitrile.

\subsubsection{Liquid-solid regime}

Figure 4 reports the values of toluene concentration versus irradiation time for runs carried out in the presence of $\mathrm{TiO}_{2}$ Merck or Degussa P25. Toluene concentration decreased after 30 minutes of mixing in the dark indicating the occurrence of physical adsorption. $\mathrm{TiO}_{2}$ Degussa P25 adsorbed a higher amount of toluene and this finding can be attributed to its higher surface area. Figure 5 reports the concentration values of toluene and of the main intermediate products ( $p$-cresol and benzaldehyde) detected during

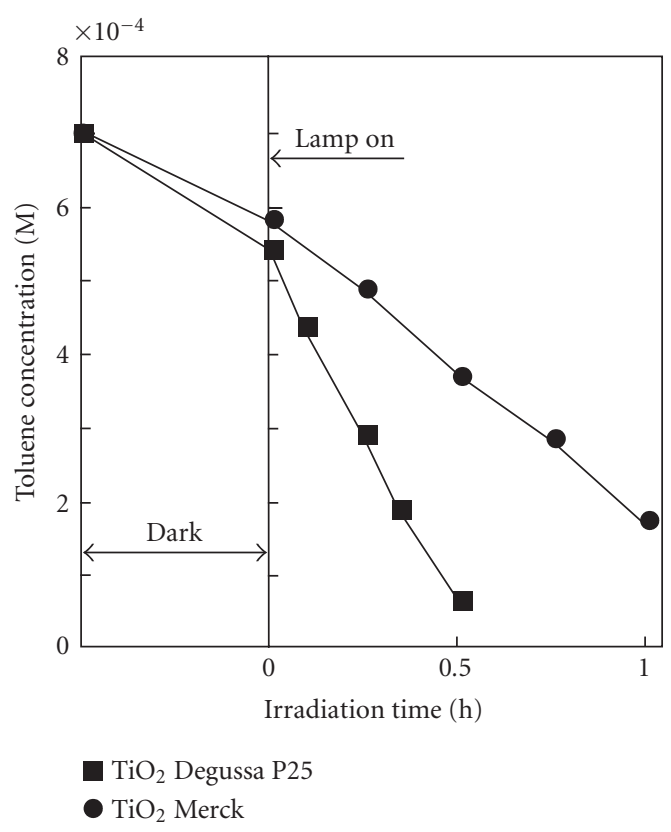

Figure 4: Toluene concentration versus irradiation time for runs carried out in batch photoreactor by using $\mathrm{TiO}_{2}$ Degussa P25 and $\mathrm{TiO}_{2} \operatorname{Merck}(\bullet)$.

a representative photodegradation run. It is worth noting that the slopes at zero time of lines interpolating the data of intermediates concentrations are different from zero. This feature clearly indicates that $p$-cresol and benzaldehyde are produced through independent reactions in parallel involving toluene. The slope of toluene disappearance plot at zero time is quite higher than the sum of slopes of the plots relative to the intermediates production. This finding indicates that there is a third pathway, in parallel with the previous ones, by which toluene is transformed to the main oxidation product detected, that is, $\mathrm{CO}_{2}$.

Figure 6 reports the values of the initial reaction rate, referred to the surface area of the photocatalyst, $\left(-r_{0}\right)$, versus the toluene concentration at the start of irradiation. For both 


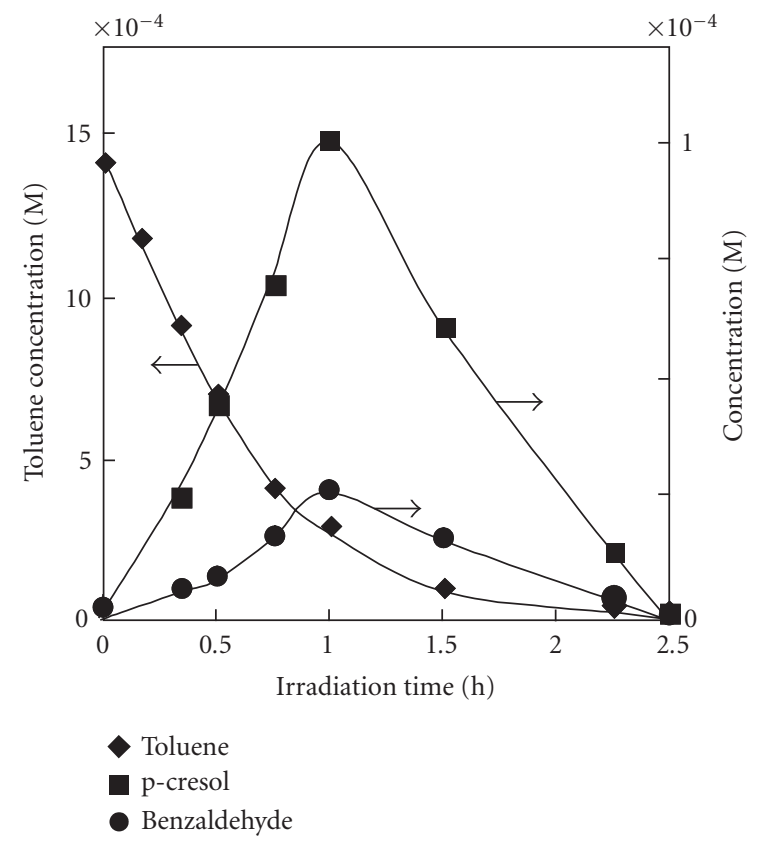

Figure 5: Toluene $(\diamond), p$-cresol $(\boldsymbol{\square})$, and benzaldehyde $(\bullet)$ concentrations versus irradiation time for a run carried out by using $\mathrm{TiO}_{2}$ Degussa P25.

catalysts, the $\left(-r_{0}\right)$ values increase by increasing the toluene concentration. In particular, the $\left(-r_{0}\right)$ values obtained in the presence of $\mathrm{TiO}_{2}$ Degussa P25 show the typical Langmuir pattern with an almost constant value for toluene concentrations higher than $0.5 \cdot 10^{-3} \mathrm{M}$, suggesting the achievement of a complete monolayer coverage. On the contrary, with $\mathrm{TiO}_{2}$ Merck, the monotone increase of $\left(-r_{0}\right)$ with the toluene concentration indicates an incomplete coverage of toluene on the catalyst surface even at the highest concentration used and the photoreactivity appears always higher with respect to that of $\mathrm{TiO}_{2}$ Degussa P25.

\subsubsection{Mechanistic aspects}

The photooxidation runs indicate that toluene degradation occurs via the formation of various intermediates, but significant differences exist due to the key role played by the nature of the interface, namely, gas-solid versus liquid-solid. As shown in Table 1, the main intermediates found in significant amounts in the liquid-solid regimes were $p$-cresol and benzaldehyde but only benzaldehyde was detected in the gassolid regime. In liquid-solid regime, traces of benzyl alcohol and pyrogallol were detected in the presence of both catalysts while benzoic acid, hydroquinone, and trans, trans muconic acid were obtained only when $\mathrm{TiO}_{2}$ Merck was used. In the gas-solid system, benzyl alcohol and benzoic acid were found adsorbed on both photocatalysts. These findings suggest the occurrence of different photodegradation pathways that depend not only on the nature of the interface but also on the nature of the $\mathrm{TiO}_{2}$ particle surface. The presence of $p$-cresol only in the liquid-solid system indicates that a high water concentration favours the attack by ${ }^{\bullet} \mathrm{OH}$ radicals to the toluene in the 4-position.

The formation of benzaldehyde and $p$-cresol and their subsequent photomineralization together with the pathway of direct photomineralization of toluene can be roughly described by the following reaction sequence:

$$
\begin{aligned}
& \mathrm{TiO}_{2}+h v \longrightarrow \mathrm{TiO}_{2}\left(e_{\mathrm{cb}}^{-}, h_{\mathrm{vb}}^{+}\right) \\
& \mathrm{O}_{2(\mathrm{ads})}+e^{-} \longrightarrow \cdot \mathrm{O}_{2(\mathrm{ads})}^{-} \\
& \cdot \mathrm{O}_{2(\mathrm{ads})}^{-}+\mathrm{H}_{2} \mathrm{O} \longrightarrow \cdot \mathrm{OOH}+\mathrm{OH}^{-} \\
& \mathrm{OH}_{(\text {surf })}^{-}+h_{\mathrm{vb}}^{+} \longrightarrow \cdot \mathrm{OH}_{(\text {surf })}
\end{aligned}
$$

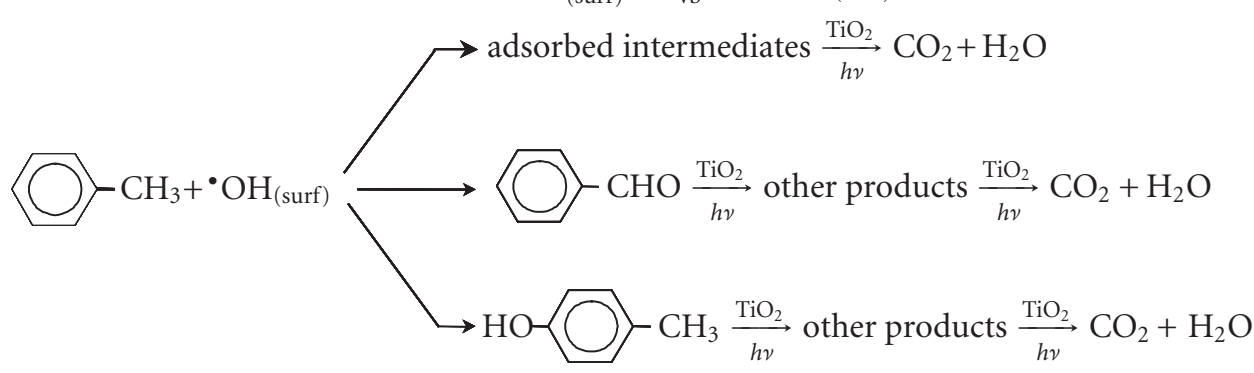

Some intermediate species detected in the liquid-solid system in the presence of $\mathrm{TiO}_{2}$ Merck were absent when $\mathrm{TiO}_{2}$ Degussa P25 was used (see Table 1). This behaviour can be explained by considering the different interactions between the active sites of the solids and the adsorbed species. As suggested by FTIR investigations [11, 12], the absence of benzoic 


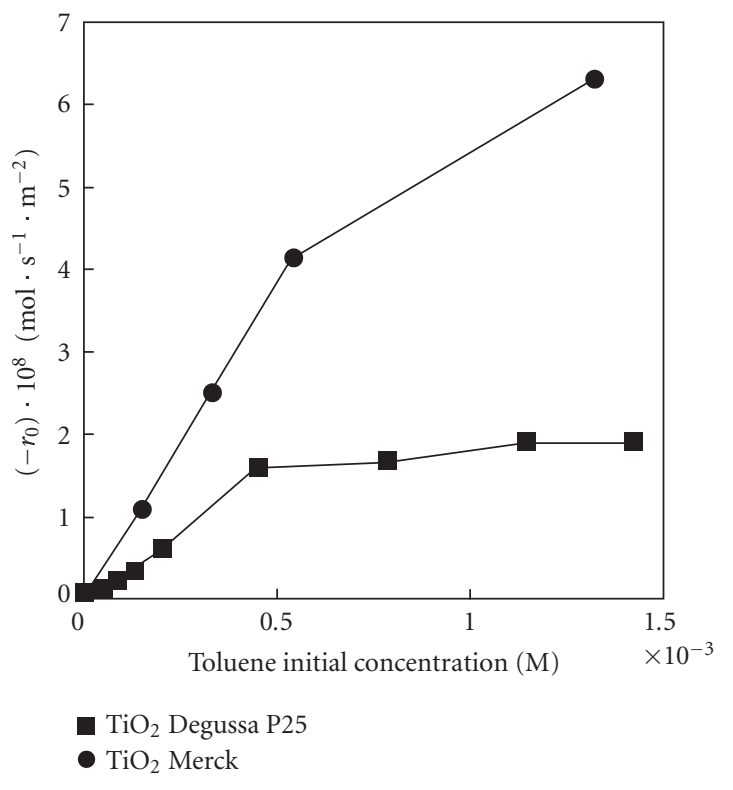

FIGURE 6: Initial reaction rate of toluene disappearance per unit surface area $\left(-r_{0}\right)$ versus initial toluene concentration. $\mathrm{TiO}_{2} \operatorname{Merck}(\bullet)$; $\mathrm{TiO}_{2}$ Degussa P25 (ם).

acid and other intermediates can be due to strong interactions (probably due to the presence of carbonylic/carboxylic groups) of these compounds with the surface of $\mathrm{TiO}_{2} \mathrm{De}-$ gussa P25 that allows the subsequent degradation of these molecules without their release into the liquid phase.

As far as the photoreactivity results obtained in the gassolid system are concerned, the deactivation of $\mathrm{TiO}_{2}$ Degussa P25, even in the presence of water vapour, can be explained by taking into account the formation of photooxidized species strongly interacting with the surface that blocks the active sites of the catalyst: adsorbed hemiacetalic species, precursors of carboxylate-like species, have been previously detected by FTIR spectroscopy [22]. The formation of unknown species cannot be excluded because of the presence of peaks (probably assignable to products with high molecular weight) in the HPLC chromatogram of the acetonitrile solution obtained by washing the photocatalyst at the end of the runs. No deactivation was observed for both catalysts in the aqueous system because the presence of liquid water not only favours the intermediate desorption but also produces the surface reconstruction, that is, the restoration of the hydroxyl groups onto the surface.

The toluene degradation rate in the liquid-solid regime (order of magnitude of $10^{-8} \mathrm{~mol} \cdot \mathrm{s}^{-1} \cdot \mathrm{m}^{-2}$ ) was $10^{4}$ times faster than that determined in the gas-solid system $\left(10^{-12} \mathrm{~mol} \cdot \mathrm{s}^{-1} \cdot \mathrm{m}^{-2}\right)$. It is known that the photodegradation kinetics is affected by the radiation intensity and by the reagent concentrations, but in the present case the photon energy impinging onto the catalyst surface was only ca. 4 times greater for the liquid-solid system with respect to the gas-solid one. As shown by the photoreactivity results, water is an essential reagent for the occurrence of reaction. The orders of magnitude of toluene and water concentrations were, respectively, $10^{-5}$ and $10^{-4} \mathrm{M}$ for the gas system, and $10^{-4}$ and $55 \mathrm{M}$ for the liquid system. Therefore, in the liquid phase, the concentration of water was about $10^{6}$ times greater than that in gas phase. It is likely that this huge difference in water concentration is responsible for the observed differences of photoreactivity between liquid-solid and gas-solid regimes, because water competes for the adsorption onto the surface sites not only with toluene but also mainly with the intermediates. Moreover, the presence of liquid water is responsible for the photoproduction because of a very higher number of oxidant radicals (see Section 3.3.1).

\subsection{Acetonitrile degradation}

\subsubsection{Gas-solid regime}

Preliminary runs were performed at increasing flow rate values with the aim of minimising mass transport resistances on the photocatalytic process. The reaction rate remained constant for flow rate values higher than ca. $1.87 \mathrm{~cm}^{3} \cdot \mathrm{s}^{-1}$ for both photocatalysts. On this basis, all the photoreactivity experiments were carried out at a flow rate of $1.87 \mathrm{~cm}^{3} \cdot \mathrm{s}^{-1}$. The determination of the acetonitrile disappearance rate per unit of catalyst surface area, $\left(-r_{S}\right)$, was performed for all of the photoreactivity runs by applying the mass balance on the whole photoreactor (see (1)).

Figure 3.2.1 shows the values of $\left(-r_{s}\right)$ obtained from runs carried out at different inlet acetonitrile concentrations. For both photocatalysts, by increasing the acetonitrile concentration at equal oxygen content, the $\left(-r_{s}\right)$ values increased up to a plateau indicating that the coverage of the surface by acetonitrile molecules increased up to the monolayer coverage. The reaction rates determined in the presence of $\mathrm{TiO}_{2}$ Merck were higher than those detected with Degussa P25, indicating that $\mathrm{TiO}_{2}$ Merck has a higher number of active sites per unit surface area where acetonitrile adsorbs and reacts with respect to Degussa P25. As shown in Figure 3.2.1, the plateau reached by increasing the inlet oxygen concentration at equal acetonitrile concentration indicates that also the oxygen coverage increases reaching the monolayer coverage.

The degradation products found in the gas phase in the presence of both photocatalysts were carbon dioxide and hydrogen cyanide while the ionic species found in the alkaline trap were cyanide ions and traces of formate. The analyses performed on the aqueous solution obtained by washing the photocatalysts at the end of the run revealed the presence of traces of nitrate and formate ions.

The values of reaction rate obtained with a dry reacting mixture were identical to those obtained in runs carried out with wet mixtures and the degradation products were the same. The photocatalysts exhibited a constant activity also for long-lasting runs (72 hours).

The amounts of hydrogen cyanide measured in the gas phase and those of produced $\mathrm{CO}_{2}$, as determined by the gravimetric method, corresponded to the moles of degraded acetonitrile. The presence of formate ions in the trap can be attributed to the partial oxidation of the organic moiety of the molecule. The very small quantities of formate and nitrate ions, found adsorbed on the surface of the used 


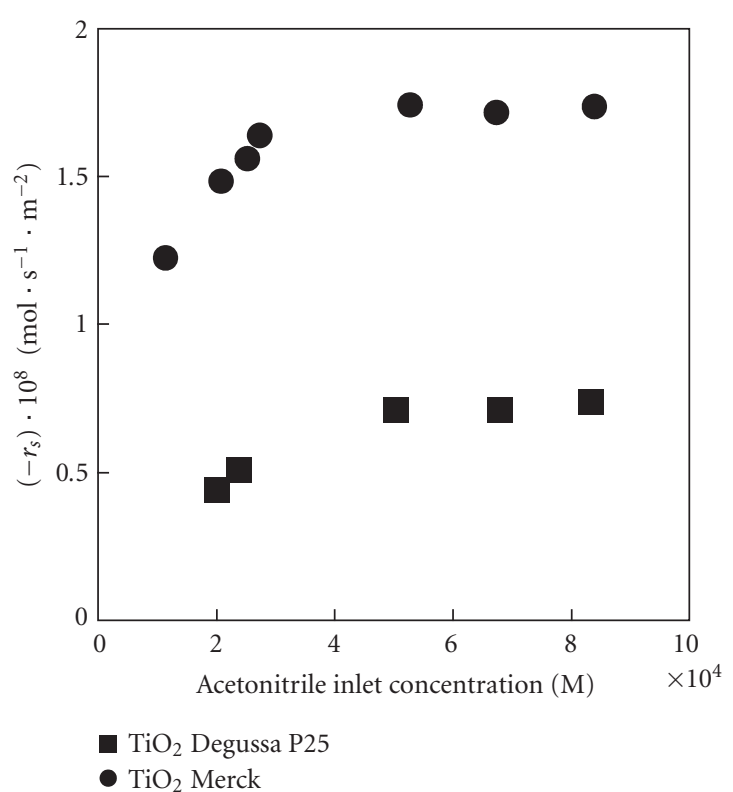

(a)

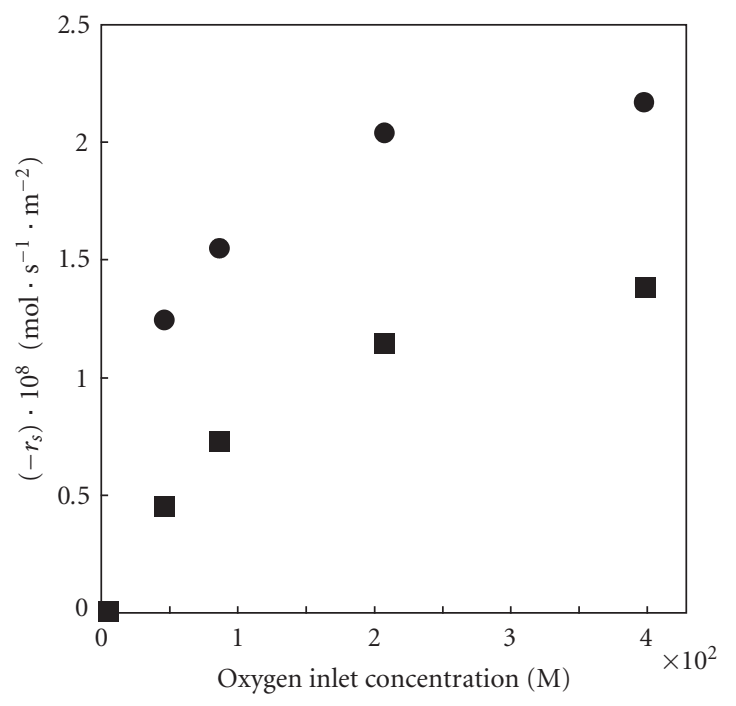

- $\mathrm{TiO}_{2}$ Degussa P25

- $\mathrm{TiO}_{2}$ Merck

(b)

FIgURE 7: Acetonitrile photocatalytic degradation in gas-solid regime: reaction rate per unit surface area, $\left(-r_{s}\right)$, versus the inlet acetonitrile concentration at $\left[\mathrm{O}_{2}\right]=1 \cdot 10^{-2} \mathrm{M}$ (a) or versus the inlet oxygen concentration at $\left[\mathrm{CH}_{3} \mathrm{CN}\right]=5 \cdot 10^{-4} \mathrm{M}(\mathrm{b})$. Flow rate: $1.87 \mathrm{~cm}^{3} \cdot \mathrm{s}^{-1}$. $\mathrm{TiO}_{2} \operatorname{Merck}(\bullet) ; \mathrm{TiO}_{2}$ Degussa P25 (ם).

catalysts, confirm that reacted acetonitrile was almost completely transformed to $\mathrm{HCN}$ and $\mathrm{CO}_{2}$. These findings suggest that acetonitrile degradation proceeds through the breaking of the $\mathrm{C}-\mathrm{C}$ bond.

\subsubsection{Liquid-solid regime}

Figures 8 and 9 report acetonitrile concentration values versus irradiation time for runs carried out at different

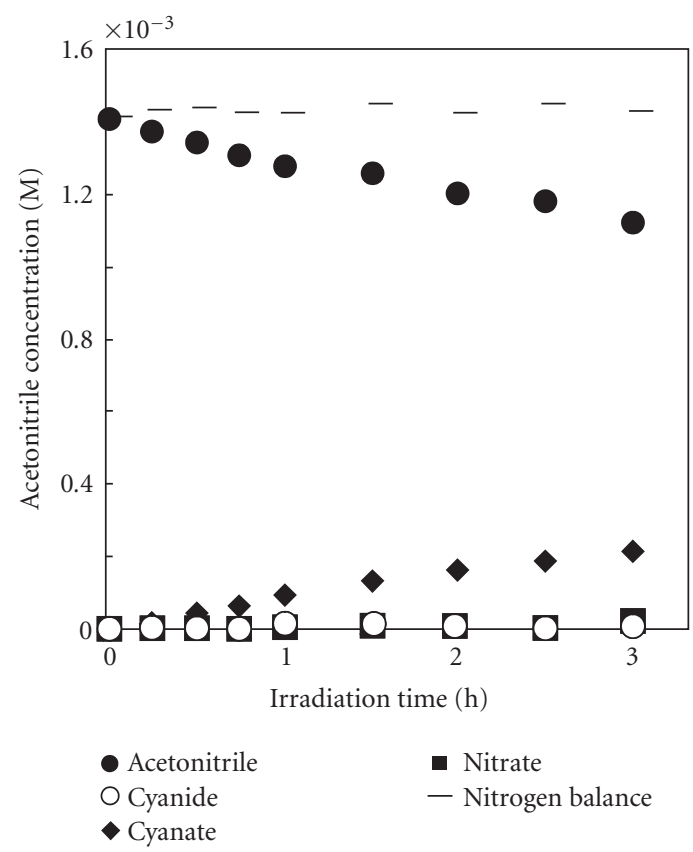

Figure 8: Acetonitrile photocatalytic degradation in liquid-solid regime: concentrations of acetonitrile $(\bullet)$, cyanide $(\bigcirc)$, cyanate $(\checkmark)$, nitrate $(\mathbf{\square})$, and nitrogen balance $(-)$ versus irradiation time. Catalyst: $\mathrm{TiO}_{2}$ Merck.

initial acetonitrile concentrations for $\mathrm{TiO}_{2}$ Merck and Degussa P25 photocatalysts, respectively. These figures also report the concentration of $\mathrm{N}$-containing ionic species, that is, cyanides, cyanates and nitrates, found in significant amounts in the reacting solution. Carbonate and small amounts of formate ions were also detected. The nitrogen molar balance, obtained taking into account the concentration of all the Ncontaining species detected in the solution, was satisfied for all the duration of the run, thus indicating that volatile nitrogen compounds were not formed in the course of acetonitrile photodegradation. This behaviour was confirmed for all the runs carried out with both photocatalysts.

The disappearance of acetonitrile in the liquid-solid regime occurs through the same pathway hypothesised in the gas-solid regime involving the surface sites of the photocatalysts able to break the C-C bond. This route transforms acetonitrile in cyanide, cyanate, nitrate, and carbonate ions. The finding that cyanate concentration in aqueous solution is far higher than that of cyanide ion since the start of acetonitrile photodegradation (see Figures 8 and 9) would suggest that the cyanide radicals, photoproduced on the catalyst surface, are predominantly transformed to cyanate instead of to cyanide ions.

\subsubsection{Mechanistic aspects}

Intermediates and reaction products revealed during the runs show that acetonitrile degradation in gas-solid and in 


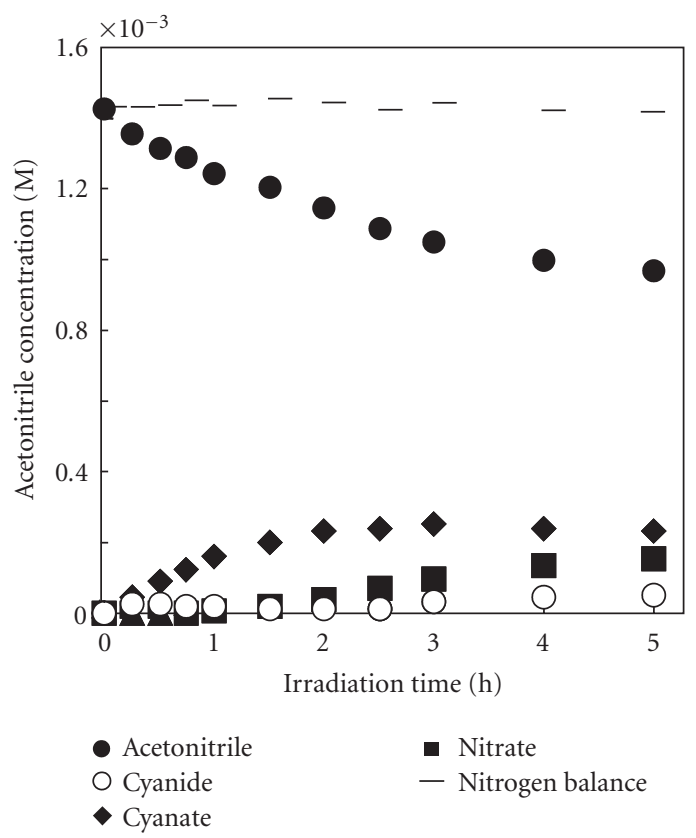

FIgURE 9: Acetonitrile photocatalytic degradation in liquid-solid regime: concentrations of acetonitrile $(\bullet)$, cyanide $(\bigcirc)$, cyanate $(\checkmark)$, nitrate $(\boldsymbol{\square})$, and nitrogen balance $(-)$ versus irradiation time. Catalyst: $\mathrm{TiO}_{2}$ Degussa P25.

liquid-solid regime proceeds through the breaking of the acetonitrile $\mathrm{C}-\mathrm{C}$ bond. The production of $\mathrm{CO}_{2}$ and $\mathrm{HCN}$ from acetonitrile oxidation in the gas-solid regime may be described by the following overall reaction:

$$
\mathrm{CH}_{3} \mathrm{CN}_{(\mathrm{ads})}+\frac{3}{2} \mathrm{O}_{2(\text { ads })} \underset{h v}{\stackrel{\mathrm{TiO}_{2}}{\longrightarrow}} \mathrm{CO}_{2}+\mathrm{HCN}+\mathrm{H}_{2} \mathrm{O}
$$

Zhuang et al. [19] claimed the breaking of the acetonitrile $\mathrm{C}-\mathrm{C}$ bond and proposed a reaction mechanism where the principal products were $\mathrm{CO}_{2}$ and $\mathrm{N}_{2}$. They observed isocyanate species on the photocatalyst surface, subsequently transformed to $\mathrm{CO}_{2}$ and $\mathrm{N}_{2}$ by the oxygen of $\mathrm{TiO}_{2}$ network. Differently from the results of the present work, the authors did not observe hydrogen cyanide, probably because this compound was not present in the adsorbed phase but it was released to the gas phase that was not analysed.

In liquid-solid regime, the acetonitrile photodegradation process produces cyanate and carbonate ions and very small amounts of cyanide, nitrate, and formate ions.

On this basis, it may be assumed that for both regimes the photocatalyst irradiation determines the acetonitrile photoadsorption $[21,23-26]$ on $\mathrm{TiO}_{2}$ surface sites. The subsequent reaction between adsorbed acetonitrile and phototoproduced $\cdot \mathrm{OH}$ radicals (see (2) to (5)) enables to break the $\mathrm{C}-\mathrm{C}$ bond producing probably cyanide radicals and methanol:

$$
\mathrm{CH}_{3} \mathrm{CN}_{(\mathrm{ads})}+{ }^{\bullet} \mathrm{OH} \rightarrow \mathrm{CH}_{3} \mathrm{OH}+\mathrm{CN}^{\bullet} .
$$

In gas solid regime, the $\mathrm{CN}^{\bullet}$ radicals react with water adsorbed on the photocatalyst surface giving rise to the formation of $\mathrm{HCN}$ that desorbs from the surface to the gas phase; methanol is photooxidised onto the surface to $\mathrm{CO}_{2}$ :

$$
\begin{array}{r}
\mathrm{CN}^{\bullet}+\mathrm{H}_{2} \mathrm{O} \longrightarrow \mathrm{HCN}+{ }^{\bullet} \mathrm{OH} \\
\mathrm{CH}_{3} \mathrm{OH}+6^{\bullet} \mathrm{OH} \longrightarrow \mathrm{CO}_{2}+5 \mathrm{H}_{2} \mathrm{O}
\end{array}
$$

Differently, in liquid-solid regime, the majority of cyanide radicals are transformed into cyanate ions which desorb from the surface to the aqueous solution; the remaining part of cyanide radicals produces cyanide ions which are also released into the solution:

$$
\begin{aligned}
\mathrm{CN}^{\bullet}+2 \mathrm{OH}^{-}+h^{+} & \longrightarrow \mathrm{CNO}^{-}+\mathrm{H}_{2} \mathrm{O} \\
\mathrm{CN}^{\bullet}+\mathrm{OH}^{-} & \longrightarrow \mathrm{CN}^{-}+{ }^{\bullet} \mathrm{OH} \\
\mathrm{CN}^{\bullet}+e_{\mathrm{C} B}^{-} & \longrightarrow \mathrm{CN}^{-}
\end{aligned}
$$

The cyanide ions produced through reaction (14) and/or (15) undergo photocatalytic oxidation to cyanate. The process continues with cyanate photooxidation steps giving rise to formation of carbonate and nitrate ions [27]:

$$
\mathrm{CNO}^{-}+10 \mathrm{OH}^{-}+8 h^{+} \longrightarrow \mathrm{CO}_{3}^{2-}+\mathrm{NO}_{3}^{-}+5 \mathrm{H}_{2} \mathrm{O}
$$

Formate and carbonate ions could be formed by the oxidation of methanol, a species not detected probably owing to its fast oxidation:

$$
\begin{gathered}
\mathrm{CH}_{3} \mathrm{OH}+2^{\bullet} \mathrm{OH} \longrightarrow \mathrm{HCHO}+2 \mathrm{H}_{2} \mathrm{O} \\
\mathrm{HCHO}+3 \mathrm{OH}^{-}+2 h^{+} \longrightarrow \mathrm{HCOO}^{-}+2 \mathrm{H}_{2} \mathrm{O} \\
\mathrm{HCOO}^{-}+3 \mathrm{OH}^{-}+2 h^{+} \longrightarrow \mathrm{CO}_{3}^{2-}+2 \mathrm{H}_{2} \mathrm{O}
\end{gathered}
$$

The orders of magnitude of the acetonitrile photodegradation rate both in liquid-solid and in gas-solid regimes were $10^{-8} \mathrm{~mol} \cdot \mathrm{s}^{-1} \cdot \mathrm{m}^{-2}$ and $10^{-9} \mathrm{~mol} \cdot \mathrm{s}^{-1} \cdot \mathrm{m}^{-2}$ in the presence of $\mathrm{TiO}_{2}$ Merck and Degussa P25, respectively. This finding confirms that the presence of water does not significantly affect the reaction rate for acetonitrile degradation, although it is worth noting that a slightly greater reaction rate was observed in gas-solid regime.

\subsection{The role of water}

The role of water during the photocatalytic degradation of toluene and acetonitrile can be elucidated by the knowledge of the nature and evolution of species present onto the catalyst surface both in the absence and in the presence of nearUV radiation.

\subsubsection{Toluene}

FT-IR investigations have shown that the adsorption of toluene on the $\mathrm{TiO}_{2}$ surface involves the $\pi$ electrons of the aromatic compound [28].

In gas-solid regime, the photodegradation of toluene produces mainly carbon dioxide and small amounts of benzaldehyde. As revealed by adsorption and desorption experiments the interaction of benzaldehyde with the surface of the 
photocatalyst significantly depends on the morphology and surface structure of the $\mathrm{TiO}_{2}$ samples $[28,29]$.

On $\mathrm{TiO}_{2}$ Merck, benzaldehyde is weakly adsorbed through hydrogen bonding with surface hydroxyl groups and it is easily released to the gas phase in the presence of water vapour [12]. When a dry reacting mixture is used, benzaldehyde is not able to desorb from the catalyst surface; its subsequent transformation determines an irreversible dehydroxylation and, as a consequence, the catalyst deactivation. Differently, on $\mathrm{TiO}_{2}$ Degussa P25, benzaldehyde is retained also in the presence of water vapour since it is transformed to hemiacetalic species by nucleophilic attack of the hydroxyl groups to the carbonyl moiety of benzaldehyde. These hemiacetalic species are successively converted to benzoic acid-like species, strongly adsorbed on the active $\mathrm{TiO}_{2}$ surface sites [22], which progressively deactivate the catalyst.

In liquid-solid regime, the high amount of water hinders the adsorption of toluene through the $\pi$ electrons so that part of toluene is adsorbed by means of the methyl group of the molecule. Differently from the reactions occurring in gas-solid regime, the direct mineralization of the aromatic ring decreases while the partial oxidation of toluene increases as indicated by the finding that benzyl alcohol, benzoic acid, and aromatic polyhydroxylate species, that is, paracresol, pyrogallol and hydroquinone, are detected in the aqueous solution in addition to benzaldehyde. This finding indicates that the water molecules, owing to their high concentration, are able both to displace the partial oxidation products of toluene from the photocatalyst surface before their complete oxidation and to reconstruct the surface hydroxyl groups consumed in the course of the photoreaction. As a consequence, no deactivation is observed in the liquid-solid systems.

\subsubsection{Acetonitrile}

The adsorption of acetonitrile on $\mathrm{TiO}_{2}$ Merck occurs by means of hydrogen bonding with the surface $\mathrm{OH}$ groups through the lone-pairs of the nitrogen atoms [21]. The interaction is weak so that acetonitrile adsorption results are completely reversible although the organic molecule is adsorbed more strongly than water on $\mathrm{TiO}_{2}$ surface.

In the case of $\mathrm{TiO}_{2}$ Degussa P25, the adsorption of the acetonitrile molecules occurs on surface $\mathrm{Ti}^{4+}$ ions with the displacement of water molecules originally coordinated to such sites. A modest amount of acetonitrile molecules, initially adsorbed on $\mathrm{Ti}^{4+}$ cations, react with adjacent $\mathrm{O}^{2-}$ anions (or $\mathrm{OH}$ groups) and form acetamide-like species that are irreversibly adsorbed on the surface also in the presence of water vapour [21].

Under irradiation, cyanate, isocyanate, formate-like species, carbonate, hydrogen carbonate, and nitrate species appear, resulting from the photooxidation of the acetonitrile molecules coordinated to $\mathrm{Ti}^{4+}$ ions. This behaviour indicates that the acetamide-like species are less sensitive to the photooxidation than acetonitrile adsorbed in molecular form, exhibiting a poisoning effect towards a small part of the surface sites. This finding accounts for the lower initial reaction rate observed for $\mathrm{TiO}_{2}$ Degussa $\mathrm{P} 25$ with respect to $\mathrm{TiO}_{2}$ Merck.

In gas-solid regime, the surface sites of both catalysts maintain their activity during the photocatalytic reaction and do not deactivate also in the absence of water vapour. In liquid-solid regime, the high concentration of water hinders the adsorption of the acetonitrile molecules onto the photocatalyst surface and this effect accounts for the slight decrease of the reaction rate with respect to that measured in the gas-solid regime. In the case of toluene degradation, the hindering effect of water molecules in liquid-solid regime is not noticed because the reaction rate in gas-solid regime is far less than that observed in liquid-solid one, due to the deactivation of the catalyst surface.

The presence of the same intermediate products confirms that the photodegradation mechanism of acetonitrile is similar in both regimes. High concentrations of water allow that the nitrile radicals, formed after the primary hydroxyl radical attack to the acetonitrile molecule, evolve to cyanide or cyanate anions that are eventually oxidised to nitrate and carbonate.

\section{CONCLUSIONS}

The photocatalytic degradation of toluene and acetonitrile successfully occurred both in gas-solid and in liquid-solid regimes by using $\mathrm{TiO}_{2}$ Degussa $\mathrm{P} 25$ or $\mathrm{TiO}_{2}$ Merck. Various intermediates were detected during toluene degradation but significant differences exist between the gas-solid and the liquid-solid systems. Carbon dioxide was the principal product in both systems; however, only benzaldehyde was revealed as intermediate in gas-solid regime whereas in liquid-solid regime the main intermediates found in significant amounts were $p$-cresol and benzaldehyde. In liquidsolid regime, traces of benzyl alcohol and pyrogallol were detected for both catalysts whereas benzoic acid, hydroquinone, and trans, trans muconic acid were found only with $\mathrm{TiO}_{2}$ Merck. These findings suggest the occurrence of different photodegradation pathways depending not only on the interfaces but also on the specific features of the photocatalyst. Liquid water played a fundamental role favouring the partial photooxidation of the molecule of toluene. Indeed the presence of liquid water not only favours the intermediate desorption but also it produces restoration of the surface hydroxyl groups.

The photocatalytic degradation of acetonitrile produced hydrogen cyanide and carbon dioxide in gas-solid regime, and also carbonate and nitrate ions in liquid-solid regime. These findings indicate that the degradation pathway started in both regimes with the breaking of the acetonitrile C-C bond. In gas-solid regime, the nitrile moiety formed cyanide radical that evolved to volatile hydrogen cyanide, whereas the methyl moiety underwent a fast and quantitative oxidation to $\mathrm{CO}_{2}$ and $\mathrm{H}_{2} \mathrm{O}$. In liquid-solid regime, the nitrile part of the molecule of acetonitrile formed cyanate, which was released to the aqueous solution undergoing subsequent photocatalytic oxidation to nitrate and carbonate, whereas the oxidation of the methyl moiety produced carbonate ion. For 
both regimes, the acetonitrile degradation rates were of the same order of magnitude, indicating that water of reaction environment does not play a significant role in the kinetics of the photoprocess.

\section{ACKNOWLEDGMENTS}

The Authors wish to thank the MIUR (Rome) for financial support. This work has been carried out in the framework of the Interuniversity Consortium "Chemistry for the Environment" (INCA, Italy).

\section{REFERENCES}

[1] M. Schiavello, Ed., Heterogeneous Photocatalysis, John Wiley \& Sons, New York, NY, USA, 1995.

[2] N. Serpone and E. Pelizzetti, Eds., Photocatalysis: Fundamentals and Applications, John Wiley \& Sons, New York, NY, USA, 1989.

[3] A. Fujishima, K. Hashimoto, and T. Watanabe, $\mathrm{TiO}_{2}$ Photocatalysis: Fundamentals and Applications, BKC, Tokyo, Japan, 1999.

[4] J. Peral and D. F. Ollis, "Heterogeneous photocatalytic oxidation of gas-phase organics for air purification: acetone, 1butanol, butyraldehyde, formaldehyde, and $m$-xylene oxidation," Journal of Catalysis, vol. 136, no. 2, pp. 554-565, 1992.

[5] M. L. Sauer and D. F. Ollis, "Photocatalyzed oxidation of ethanol and acetaldehyde in humidified air," Journal of Catalysis, vol. 158, no. 2, pp. 570-582, 1996.

[6] A. L. Linsebigler, G. Lu, and J. T. Yates Jr, "Photocatalysis on $\mathrm{TiO}_{2}$ surfaces: principles, mechanisms, and selected results," Chemical Reviews, vol. 95, no. 3, pp. 735-758, 1995.

[7] T. Ibusuki and K. Takeuchi, "Toluene oxidation on u.v.irradiated titanium dioxide with and without $\mathrm{O}_{2}, \mathrm{NO}_{2}$ or $\mathrm{H}_{2} \mathrm{O}$ at ambient temperature," Atmospheric Environment, vol. 20, no. 9, pp. 1711-1715, 1986.

[8] T. N. Obee and R. T. Brown, " $\mathrm{TiO}_{2}$ photocatalysis for indoor air applications: effects of humidity and trace contaminant levels on the oxidation rates of formaldehyde, toluene, and 1,3-butadiene," Environmental Science \& Technology, vol. 29, no. 5, pp. 1223-1231, 1995.

[9] Y. Luo and D. F. Ollis, "Heterogeneous photocatalytic oxidation of trichloroethylene and toluene mixtures in air: kinetic promotion and inhibition, time-dependent catalyst activity," Journal of Catalysis, vol. 163, no. 1, pp. 1-11, 1996.

[10] H. Einaga, S. Futamura, and T. Ibusuki, "Heterogeneous photocatalytic oxidation of benzene, toluene, cyclohexene and cyclohexane in humidified air: comparison of decomposition behavior on photoirradiated $\mathrm{TiO}_{2}$ catalyst," Applied Catalysis B: Environmental, vol. 38, no. 3, pp. 215-225, 2002.

[11] V. Augugliaro, S. Coluccia, V. Loddo, et al., "VOC's abatement: photocatalytic oxidation of toluene in vapour phase on anatase $\mathrm{TiO}_{2}$ catalyst," Studies in Surface Science and Catalysis, vol. 110, pp. 663-672, 1997.

[12] G. Martra, V. Augugliaro, S. Coluccia, et al., "Photocatalytic oxidation of gaseous toluene on polycrystalline $\mathrm{TiO}_{2}$ : FT-IR investigation of surface reactivity of different types of catalyst," Studies in Surface Science and Catalysis, vol. 130, pp. 665-670, 2000.
[13] M. Fujihira, Y. Satoh, and T. Osa, "Heterogeneous photocatalytic oxidation of aromatic compounds on $\mathrm{TiO}_{2}$," Nature, vol. 293, pp. 206-208, 1981.

[14] M. Fujihira, Y. Satoh, and T. Osa, "Heterogeneous photocatalytic reactions on semiconductor materials: Part II. Photoelectrochemistry at semiconductor $\mathrm{TiO}_{2} /$ insulating aromatic hydrocarbon liquid interface," Journal of Electroanalytical Chemistry, vol. 126, no. 1-3, pp. 277-281, 1981.

[15] J. A. Navio, M. García Gómez, M. A. Pradera Adrian, and J. Fuentes Mota, "Partial or complete heterogeneous photocatalytic oxidation of neat toluene and 4-picoline in liquid organic oxygenated dispersions containing pure or iron-doped titania photocatalysts," Journal of Molecular Catalysis A: Chemical, vol. 104, no. 3, pp. 329-339, 1996.

[16] V. Augugliaro, V. Loddo, G. Marcì, et al., "Photocatalytic degradation of toluene in aqueous suspensions of polycrystalline $\mathrm{TiO}_{2}$ in the presence of the surfactant tetradecyldimethylamino-oxide," Studies in Surface Science and Catalysis, vol. 130, pp. 1973-1978, 2000.

[17] G. Marcí, M. Addamo, V. Augugliaro, et al., "Photocatalytic oxidation of toluene on irradiated $\mathrm{TiO}_{2}$ : comparison of degradation performance in humidified air, in water and in water containing a zwitterionic surfactant," Journal of Photochemistry and Photobiology A: Chemistry, vol. 160, no. 1, pp. 105114, 2003.

[18] N. N. Lichtin and M. Avudaithai, " $\mathrm{TiO}_{2}$-photocatalyzed oxidative degradation of $\mathrm{CH}_{3} \mathrm{CN}, \mathrm{CH}_{3} \mathrm{OH}, \mathrm{C}_{2} \mathrm{HCl}_{3}$, and $\mathrm{CH}_{2} \mathrm{Cl}_{2}$ supplied as vapors and in aqueous solution under similar conditions," Environmental Science \& Technology, vol. 30, no. 6, pp. 2014-2020, 1996.

[19] J. Zhuang, C. N. Rusu, and J. T. Yates Jr, "Adsorption and photooxidation of $\mathrm{CH}_{3} \mathrm{CN}$ on $\mathrm{TiO}_{2}$," The Journal of Physical Chemistry. B, vol. 103, no. 33, pp. 6957-6967, 1999.

[20] V. Augugliaro, A. Bianco Prevot, J. Cáceres Vázquez, et al., "Photocatalytic oxidation of acetonitrile in aqueous suspension of titanium dioxide irradiated by sunlight," Advances in Environmental Research, vol. 8, no. 3-4, pp. 329-335, 2004.

[21] P. Davit, G. Martra, S. Coluccia, et al., "Adsorption and photocatalytic degradation of acetonitrile: FT-IR investigation," Journal of Molecular Catalysis A: Chemical, vol. 204-205, pp. 693-701, 2003.

[22] G. Martra, "Lewis acid and base sites at the surface of microcrystalline $\mathrm{TiO}_{2}$ anatase: relationships between surface morphology and chemical behaviour," Applied Catalysis A: General, vol. 200, no. 1-2, pp. 275-285, 2000.

[23] J. Cunningham, M. Jauch, and D. McNamara, "Adsorbate activation on metal oxides by single-electron and electron-pair interactions," Proceedings of the Royal Irish Academy. Section B: Biological, Geological, and Chemical Science, vol. 89, pp. 299320, 1989.

[24] K. Hadjiivanov, D. Klissurski, G. Busca, and V. Lorenzelli, "Benzene-ammonia coadsorption on $\mathrm{TiO}_{2}$ (anatase)," Journal of the Chemical Society, Faraday Transactions, vol. 87, no. 1, pp. 175-178, 1991.

[25] R. I. Bickley, Heterogeneous Photocatalysis, vol. 3, John Wiley \& Sons, New York, NY, USA, 1997.

[26] C.-C Chuang, W.-C. Wu, M.-X. Lee, and J.-L. Lin, "Adsorption and photochemistry of $\mathrm{CH}_{3} \mathrm{CN}$ and $\mathrm{CH}_{3} \mathrm{CONH}_{2}$ on powdered $\mathrm{TiO}_{2}$," Physical Chemistry Chemical Physics, vol. 2, no. 17, pp. 3877-3882, 2000. 
[27] V. Augugliaro, V. Loddo, G. Marcì, L. Palmisano, and M. J. López-Muñoz, "Photocatalytic oxidation of cyanides in aqueous titanium dioxide suspensions," Journal of Catalysis, vol. 166, no. 2, pp. 272-283, 1997.

[28] M. Nagao and Y. Suda, "Adsorption of benzene, toluene, and chlorobenzene on titanium dioxide," Langmuir, vol. 5, no. 1, pp. 42-47, 1989.

[29] G. Martra, S. Coluccia, L. Marchese, et al., "The role of $\mathrm{H}_{2} \mathrm{O}$ in the photocatalytic oxidation of toluene in vapour phase on anatase $\mathrm{TiO}_{2}$ catalyst: a FTIR study," Catalysis Today, vol. 53, no. 4, pp. 695-702, 1999. 


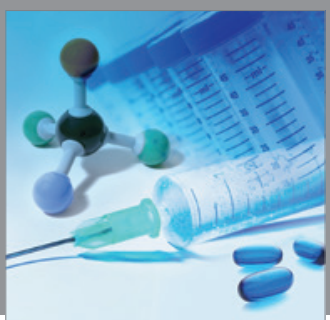

International Journal of

Medicinal Chemistry

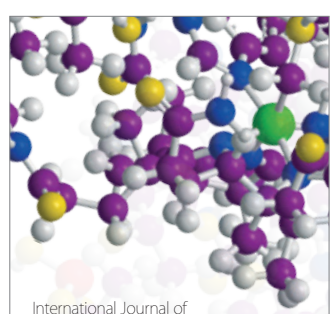

Carbohydrate Chemistry

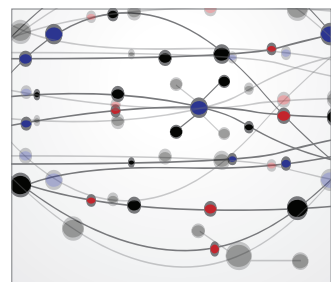

The Scientific World Journal
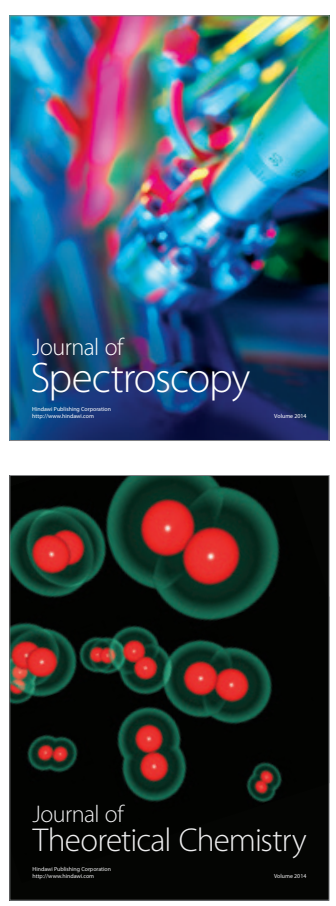
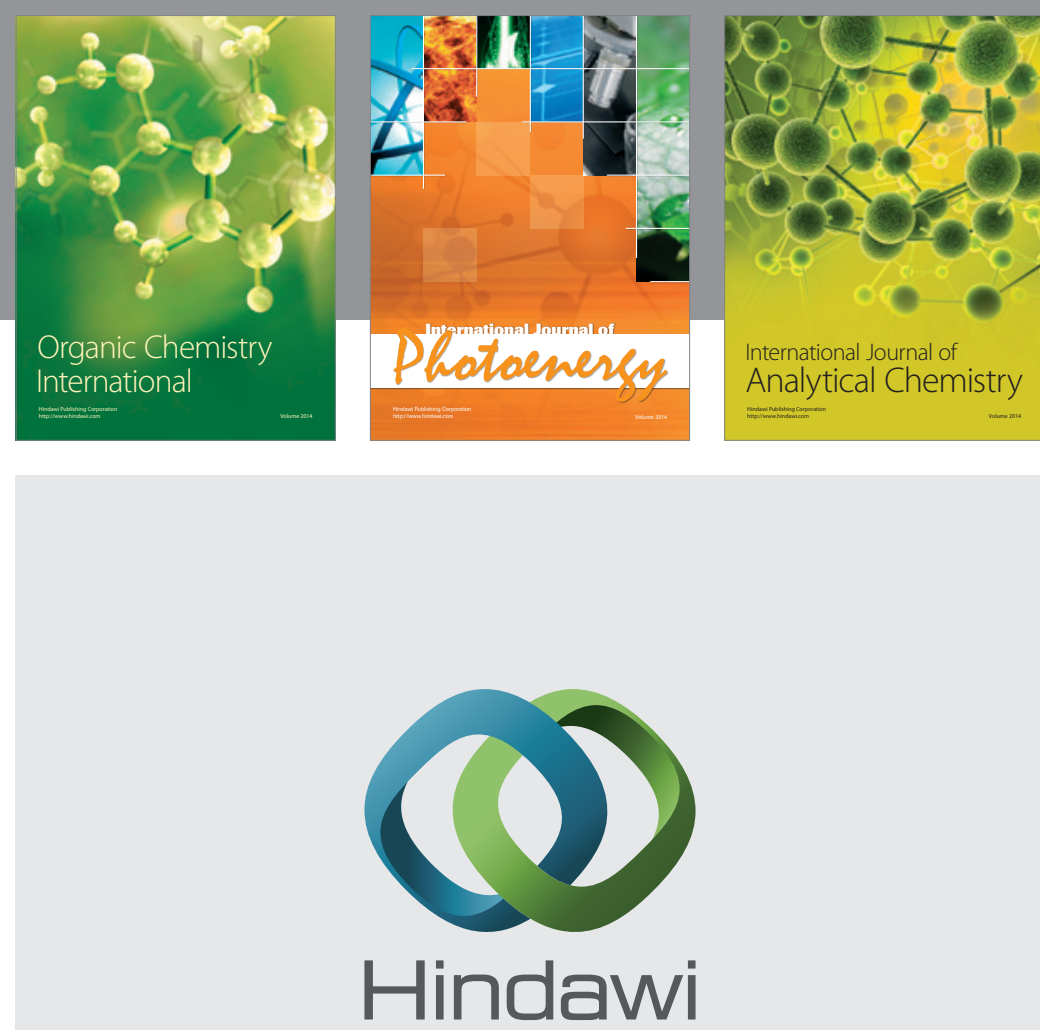

Submit your manuscripts at

http://www.hindawi.com
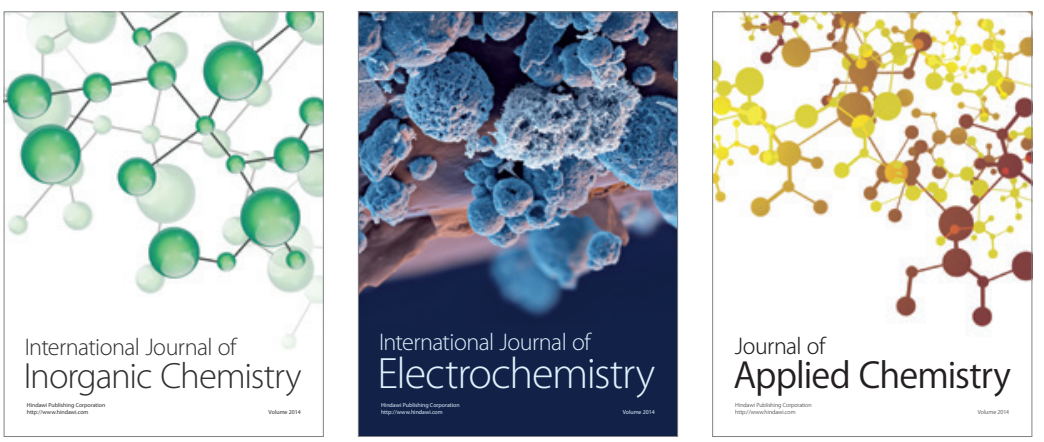

Journal of

Applied Chemistry
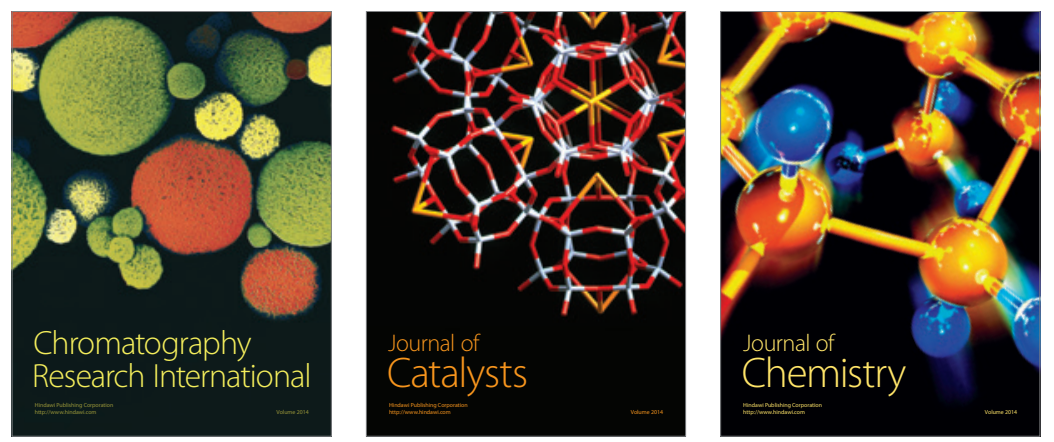
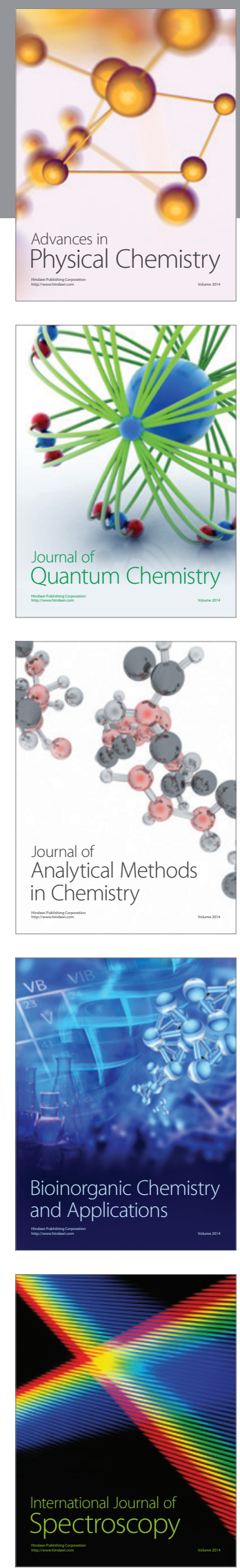